Rev. SINAPSIS, Vol. 3, Nº 2, Diciembre 2013

ISSN $1390-7832$

\title{
El proceso de enseñanza aprendizaje del inglés para el estudiante de la carrera de Comercio Exterior
}

\section{La enseñanza del inglés para el comercio exterior}

Maritza Sandra Pibaque Ponce, Mg. DUIE. ${ }^{(1)}$

Ned Quevedo Arnaiz Dr. C. ${ }^{(2)}$

${ }^{(1)}$ Universidad Estatal del Sur de Manabí, Ecuador

${ }^{(2)}$ Universidad de Camagüey Ignacio Agramante Loynaz, Cuba

Contacto: mimbaque@gmail.com

Receptado: 03/08/2013_Aceptado: 04/10/2013

\section{Resumen}

La disciplina del idioma inglés es parte de las mallas curriculares en el nivel universitario con el objetivo de posibilitar la comunicación en la lengua inglesa con la integración de las cuatro habilidades (expresión oral, audición, lectura y escritura) para que le sea útil en la vida laboral del profesional. Este trabajo se traza como objetivo caracterizar el proceso de enseñanza aprendizaje del inglés para estudiantes de la carrera de Comercio Exterior para el desarrollo de la competencia comunicativa en dicho proceso de enseñanza aprendizaje del inglés en la Universidad. Para alcanzar los resultados esperados se han utilizado diferentes métodos para conocer la esencia del fenómeno analizado. Así, entre otros para el desarrollo de la investigación han sido fundamentales los siguientes métodos: Análisis y síntesis, Inductivo-deductivo y el Método sistémico funcional. Los procesos de enseñanza y aprendizaje promueven en cada estudiante la apropiación del conocimiento, pero el proceso en sí influye significativamente en las formas de aprendizaje, y las relaciones que establece el profesional con el entorno del Comercio Exterior.

Palabras claves: Enseñanza- aprendizaje, competencias, construcción del conocimiento, aprendizaje de lenguas

Prevalence of teenage pregnancy and its psychosocial impact on the general hospital served Jipijapa, Ecuador 


\begin{abstract}
The discipline of English is part of the curricula at the university level in order to enable communication in the English language with the integration of the four skills (speaking, listening, reading and writing) to make it useful in professional working life. This work is drawn to characterize the process of teaching learning English for students of the career Foreign Trade for the development of communicative competence in the teaching-learning process of English at the University. To achieve the expected results have used different methods to learn the essence of the phenomenon analyzed. Thus, among others for the development of fundamental research have been the following methods: Analysis and synthesis, inductive-deductive and systemic
\end{abstract}

functional method. The processes of teaching and learning in each student promote the appropriation of knowledge, but the process itself significantly influences the ways of learning, and the professional relationship with the external trade environment.

Keywords: Teaching - learning, competences, knowledge, language learning

\title{
Introducción
}

En el complejo contexto de globalización que hoy tiene lugar a escala mundial, reviste particular importancia la educación, para esclarecer posiciones y defender la identidad cultural y nacional de cada pueblo. Mediante la educación el ser humano se apropia críticamente de los avances culturales de la humanidad, y con la educación se atiende a las diferencias culturales de cada región.

La disciplina del idioma inglés es parte de las mallas curriculares en el nivel universitario con el objetivo de posibilitar la comunicación en la lengua inglesa con la integración de las cuatro habilidades (expresión oral, audición, lectura y escritura) para que le sea útil en la vida laboral, es por ello, que el estudio del inglés permite al estudiante demostrar estas habilidades para comunicarse de forma elemental (oral y escrita) en temas relevantes de su especialidad.

Este trabajo se traza como objetivo caracterizar el proceso de enseñanza aprendizaje del inglés para estudiantes de la carrera de Comercio Exterior para el desarrollo de la competencia comunicativa en dicho proceso de enseñanza aprendizaje del inglés en la Universidad. 
Para alcanzar los resultados esperados se han utilizado diferentes métodos para conocer la esencia del fenómeno analizado. Así, entre otros para el desarrollo de la investigación han sido fundamentales los siguientes métodos: Análisis y síntesis: necesario para la revisión bibliográfica, de manera que se actualicen los conocimientos teóricos para la investigación. Inductivo-deductivo: importante para la determinación de los elementos relacionados con la conceptualización del proceso de enseñanza aprendizaje en idioma inglés. Método sistémico funcional: necesario para descubrir los nexos esenciales de los diferentes paradigmas con la enseñanza de lenguas y al desarrollo de la comunicación oral y la importancia del idioma extranjero inglés para la formación del profesional de Comercio Exterior.

\section{Desarrollo}

La Dra.C. R. Antich, (1986) plantea la idea de que el estudiante transita en su aprendizaje de una segunda lengua desde aprovechamiento de lo conocido en su lengua materna hasta lo nuevo en la lengua que aprende: "En el aprendizaje de lenguas extranjeras el estudiante trata de encontrar el equivalente en su lengua materna del texto escuchado (o leído) en la lengua extranjera". Esto va disminuyendo en la medida que comienzan a trabajar con propiedad en la lengua extranjera para conformar un sistema integrado único, es decir, cuando el estudiante comienza a pensar en la lengua extranjera.

El aprendizaje como categoría de la pedagogía ha transitado por varios momentos importantes en su conceptualización, lo cual ha influido la enseñanza de lenguas. El aprendizaje se concibe de diferentes formas según el paradigma con el que se aprecie este proceso. De ellos el primero y que más huellas ha dejado en la enseñanza es el paradigma conductista.

El enfoque conductista tuvo sus orígenes en las primeras décadas del Siglo XX, justo cuando se comenzó el auge de la enseñanza y aprendizaje del inglés como lengua extranjera. Fue J. B. Watson su fundador, quien expuso su programa y concepción a partir de un texto seminal escrito por él en 1913 denominado "La psicología desde el punto de vista conductista". Watson, de formación funcionalista (Escuela de Chicago), mostró un nuevo planteamientto teóricometodológico influido por: una concepción fixista del darwinismo, el empirismo inglés, la 
Rev. SINAPSIS, Vol. 3, No 2, Diciembre 2013

ISSN $1390-7832$

filosofía pragmatista y la concepción positivista de la ciencia (Chaplin y Krawicc, 1979; Yaroshevsky, 1979).

El conductismo en sus inicios se valoró como revolucionario porque rompía con muchos de los esquemas precedentes. El planteamiento watsoniano tuvo un buen acogimiento en la enseñanza y aprendizaje de lenguas; pero, el conductismo de Watson rápidamente se diversificó, contándose con un grupo numeroso de escuelas disímiles entre sí a partir de la década del 20 (Rubinstein, 1974). Algunos años después se desarrolló el movimiento conocido como neoconductista, con cuatro derivaciones que también se reflejaron en la enseñanza de lenguas extranjeras: el conductismo asociacionalista de E. Guthrie, el conductismo metodológico de C. L. Hull, el conductismo intencional de E. L. Tolman y finalmente el conductismo operante de B. F. Skinner.

Este último ganó importancia en la enseñanza de lenguas entre los años 40 y 60 hasta la aparición de un artículo de Chomsky criticando sus postulados basados en la formula lineal de estímulo y respuesta. La propuesta skinneriana, también llamada Análisis Experimental de la Conducta (AEC), se ha caracterizado por hacer una feroz defensa de los aspectos más radicales de la corriente conductista (el antimentalismo y el ambientalismo extremo). Según el autor norteamericano, la conducta de los organismos es explicada a través de las contingencias ambientales, negándose toda posibilidad causal-explicativa a los procesos internos de naturaleza mental, corriente que se desarrollaría después.

El alumno de lenguas, a pesar de que los conductistas concebían y deseaban promover un alumno activo, resultaba obvio que tenía un nivel de actividad fuertemente restringida por la actitud del profesor-programador, que se instituía incluso antes de la situación instruccional. La participación del alumno, por tanto estaba condicionada por las características de los contenidos del programa de enseñanza utilizado para aprender y los métodos con que se impartía.

El siguiente paradigma que influyó la enseñanza de lenguas estuvo basado en la Psicología Cognitiva. No cabe duda que la Psicología Cognitiva está relacionada con la génesis y el desarrollo del enfoque del procesamiento de información en Norteamérica, a partir de los 
Rev. SINAPSIS, Vol. 3, Nº 2, Diciembre 2013

ISSN $1390-7832$

avances que en la enseñanza de lenguas se obtuvo después de la guerra con el método del ejército.

El enfoque del procesamiento de información, considerado como parte fundamental de la Psicología Cognitiva, tiene un gran caudal de implicaciones y aplicaciones al campo de la

educación. El enfoque del procesamiento de la información, según varios autores (De Vega, 1984; Gardner, 1987; Pozo, 1989) apareció a finales de la década de los cincuenta. En esos años surgieron investigaciones de naturaleza seminal, en el campo de la cognición. Estas investigaciones tuvieron su origen en tres grandes áreas científicas: la lingüística, la teoría de la información y la computación.

Además de los mencionados campos científicos, hay que reconocer otras dos situaciones históricas externas, que crearon una atmósfera propicia para el desarrollo del nuevo paradigma. Primero, algunos autores han mencionado la importancia de la revolución tecnológica de la postguerra en Norteamérica en el campo de las comunicaciones y la informática (Bruner, 1983, cit. por Pozo, 1989; Riviere, 1987). Segundo, en esta misma década se comenzó a generar un clima de crítica y desconfianza hacia el paradigma conductista por la impugnación directa hacia sus concepciones epistemológicas y metodológicas subyacentes, basadas en el positivismo y el fisicalismo.

A partir de los sesenta y hasta el presente, se han desarrollado un considerable número de investigaciones e información teórica sobre las distintas facetas de la cognición (entendida como la adquisición, organización y uso del conocimiento, Neisser, 1982) inspiradas en la metáfora del ordenador. Según esta metáfora, el ordenador es una instancia de los sistemas de procesamiento de información, la cual según los cognitivistas, también pertenece el hombre. El paradigma del procesamiento de la información, no es una aproximación monolítica; existen actualmente varias corrientes y tradiciones bajo este enfoque paradigmático ( De Vega, 1984; Riviere, 1989). El enfoque cognitivo está interesado en el estudio de la representación mental, considerada como un espacio de problemas propios, más allá del nivel biológico y al mismo tiempo distinto del nivel sociológico o cultural. Los teóricos del procesamiento de 
Rev. SINAPSIS, Vol. 3, N² 2, Diciembre 2013

ISSN $1390-7832$

información están interesados en describir y explicar la naturaleza de las representaciones mentales, así como el determinar el papel que juegan en la producción de las acciones y conductas humanas, entre ellas las comunicativas en una lengua extranjera.

A pesar de ciertas tendencias innatistas dentro de la corriente cognitiva (como el caso de los psicolingüistas chomskianos), más recientemente dentro del paradigma del procesamiento de información una gran cantidad de teóricos han confesado una postura constructivista en sus concepciones sobre cómo el sujeto conoce los eventos externos e internos. De acuerdo con

esta concepción, el sujeto posee una organización interna de eventos que va reelaborando, en función de los intercambios con el exterior y a partir de esta organización interna (estructuras, esquemas, reglas, etc.) el sujeto interpreta y vuelve a significar la realidad.

Dentro de la corriente cognitivista hay dos autores quienes son sin duda los pilares de una serie de propuestas para el aprendizaje de lenguas y para el aprendizaje en general hasta nuestros días: Jerome Bruner, quien planteó las múltiples facetas de la cognición (relacionado a temas como pensamiento, percepción, lenguaje, etc.), y el teórico pedagógico Davis P. Ausubel.

J. Bruner es ciertamente uno de los psicólogos cognitivos de la educación con mayor trayectoria, cuya obra causó un fuerte impacto en los sesenta y parte de los setenta en Norteamérica con sus propuestas del aprendizaje por descubrimiento y acerca del currículo para pensar. Ausubel igualmente, durante la década de los sesenta elaboró la teoría del aprendizaje significativo o de la asimilación y fue uno de los teóricos que mayor inquietud ha demostrado por el análisis multidisciplinar de los problemas educativos en contextos escolares.

El proceso de aprendizaje concebido desde la perspectiva de Ausubel, es el proceso por el cual el sujeto del aprendizaje procesa la información de manera sistemática y organizada y no solo de manera memorística sino que construye y reconstruye el conocimiento (Díaz, 1998:18).

El alumno que aprende una lengua extranjera es entendido como un sujeto activo procesador de información, que posee una serie de esquemas, planes y estrategias para aprender y solucionar problemas, los cuales a su vez deben ser desarrollados. Siempre en cualquier 
Rev. SINAPSIS, Vol. 3, N² 2, Diciembre 2013

ISSN $1390-7832$

contexto escolar, por más restrictivo que este sea existe un cierto nivel de actividad cognitiva, por lo que se considera que el alumno nunca es un ente pasivo que está a merced de las contingencias ambientales o instruccionales. Desde el punto de vista cognitivo, esta actividad inherente, debe ser desarrollada para lograr un procesamiento más efectivo. La actividad cognitiva por tanto estará presente en el aprendizaje.

Según Ausubel (1976), no todos los tipos de aprendizaje son iguales, como lo habían señalado los conductistas, para quienes sólo existe una forma de aprender. En realidad, y la práctica lo confirma, existen diferentes tipos de aprendizajes que se dan dentro del aula escolar. Para comprenderlos conviene hacer dos distinciones básicas:

1) En torno al tipo de aprendizaje realizado por el alumno (la forma en que lo incorpore dentro de su estructura cognoscitiva).

2) Respecto al tipo de estrategia de enseñanza que se siga.

De acuerdo con la primera dimensión se pueden distinguir dos modalidades de aprendizaje: el repetitivo o memorístico y el significativo; conforme a la segunda, debe distinguirse entre aprendizaje por recepción y por descubrimiento. Paradójicamente, los cuatro tipos de aprendizaje se ponen de manifiesto en la enseñanza de lenguas extranjeras y cada uno cumple su rol en el proceso.

El aprendizaje memorístico, consiste en aprender la información en forma literal o al pie de la letra, tal cual ha sido enseñada por el profesor. Un ejemplo de aprendizaje memorístico sería el aprendizaje de un número telefónico o el de un poema, o el de una función comunicativa que se repite hasta la saciedad para que se fije en el alumno.

El aprendizaje significativo, en oposición, consiste en la adquisición de la información en forma sustancial (lo esencial semánticamente) y su incorporación dentro de la estructura cognoscitiva no es arbitraria, como en el aprendizaje memorístico, sino relacionando dicha información con el conocimiento previo. Aquí el aprendizaje de lengua estaría relacionado con la situación cercana a la realidad del alumno. 
Rev. SINAPSIS, Vol. 3, No 2, Diciembre 2013

ISSN 1390 - 7832

El aprendizaje receptivo, se refiere a la adquisición de productos acabados de información, donde la participación del alumno consiste simplemente en internalizar dicha información. Este tipo de aprendizaje se suele confundir con el primero pero sin duda no son iguales, porque se puede tener aprendizaje por recepción memorístico o significativo. En el aprendizaje de lenguas el desarrollo de habilidades conlleva al alumno a interiorizar muchas reglas y normas que tienen una naturaleza receptiva sean arbitrarias o no, o sea memorísticos o no.

El aprendizaje por descubrimiento, es aquel donde el contenido principal de la información a aprender no se da en su forma final, sino que debe ser descubierta por el alumno. En este aprendizaje desde la enseñanza de lenguas se realiza negociación de significados e interacción comunicativa por lo que el alumno puede llegar a elaborar su propio conocimiento en la práctica.

Otro paradigma importante en la enseñanza y aprendizaje de lengua es el llamado constructivismo. Los orígenes del paradigma constructivista se remontan a los años 30 del siglo anterior con los primeros trabajos realizados por Jean Piaget sobre la lógica y el pensamiento verbal de los niños. Esos trabajos fueron elaborados, a partir de las inquietudes epistemológicas que el autor suizo había manifestado desde su juventud. Piaget fue biólogo de formación, pero tenía una especial predilección por problemas de corte filosófico y especialmente sobre los referidos al tópico del conocimiento. De manera que pronto le inquietó la posibilidad de elaborar una epistemología biológica o científica, dado que según él existía una continuidad entre la vida (las formas de organización orgánica) y el pensamiento (lo racional). El camino más corto para tal proyecto, según el propio Piaget, debía encontrarse en la disciplina psicológica, por lo que decidió incursionar en ella con ese objetivo.

La obra piagetana está signada por el tránsito de un nivel del conocimiento a otro. Este autor desarrolló investigaciones empíricas sobre las cuestiones epistemológicas que le interesaban, desde la continuidad de los hechos por lo que su tarea le llevaría todos los años de su vida adulta (60 años de investigaciones), con la satisfacción de haber esbozado el esqueleto de una epistemología genética. La problemática central de Piaget fue pues epistémica. 
Rev. SINAPSIS, Vol. 3, No 2, Diciembre 2013

ISSN $1390-7832$

Varios autores han intentado aplicar las ideas de Piaget en el campo de la educación. En Norteamérica, a partir de los años sesenta, comenzó a ser redescubierta la obra piagetiana, debido básicamente al "boom" existente por la búsqueda de innovaciones educativas y por la ponderación de las posibilidades inherentes de la Teoría Genética en tanto teoría epistemológica y teoría del desarrollo intelectual. Durante este período y en los años posteriores, las aplicaciones e implicaciones del paradigma al campo de la educación, comenzaron a proliferar en forma notable (especialmente en la educación básica, luego también en la educación media y finalmente en la superior), y llegaron hasta posiciones más críticas y reflexivas con una interpretación más correcta en relación al uso educativo de la teoría.

Otros autores (Coll, 1983; DeVries y Kohlberg, 1986; Kamii y DeVries, 1977; Marro, 1983), han intentado clasificaciones y realizado análisis, entre los que destacan las siguientes conclusiones: 1) no existe un solo camino en las interpretaciones o lecturas de la teoría para su utilización al campo educativo y 2) a pesar de los enormes esfuerzos realizados, existe aún mucho trabajo de investigación por hacer (especialmente en el campo de los aprendizajes de

los contenidos escolares), lo que aún no ha redundado en el gran impacto esperado del paradigma a la educación.

Por tanto hay diferencias sustanciales entre los paradigmas anteriores, basados unos en: las asociaciones, los estímulos, el reforzamiento de estos, y los otros en: el procesamiento de la información y la estructura cognoscitiva, con el constructivismo que desde sus orígenes trata de: interpretar el conocimiento, los niveles del conocimiento y sobre todo la búsqueda de ese conocimiento en las respuestas científicas e interdisciplinarias, en lo que se ha dado en llamar construcción del conocimiento.

Una categoría fundamental del paradigma constructivista para la explicación de la construcción del conocimiento son las acciones (físicas y mentales) que realiza el sujeto cognoscente frente al objeto de conocimiento. Al mismo tiempo el objeto también "actúa" sobre el sujeto o "responde" a sus acciones, promoviendo en éste cambios dentro de sus representaciones que tiene de él. Por tanto, existe una interacción recíproca entre el sujeto de conocimiento y 
Rev. SINAPSIS, Vol. 3, Nº 2, Diciembre 2013

ISSN $1390-7832$

el objeto. El sujeto transforma al objeto al actuar sobre él y al mismo tiempo estructura y transforma sus estructuras o marcos conceptuales en un constante desarrollo.

En el esquema conceptual piagetiano hay siempre que partir de la categoría de la acción. El sujeto actúa para conocer al objeto: en ello se encierra el principio fundamental de toda interacción recíproca del sujeto y el objeto de conocimiento en el proceso del conocimiento. Sin embargo, hay que señalar a la vez que dichas acciones, por más primitivas que sean, como por ejemplo: los reflejos innatos, son producto directo de un cierto patrón de organización dentro del sujeto.

Esta organización de las acciones en el sujeto cognoscente, según Piaget, se debe a los esquemas, que son precisamente los "ladrillos" de toda la construcción del sistema intelectual o cognitivo, los que regulan las interacciones del sujeto con la realidad y a su vez sirven para asimilar la nueva información, o lo que es igual adaptarlas,( la cual es facilitada por las interacciones Sujeto - Objeto). Por ello en el desarrollo cognitivo, los procesos de organización y de adaptación serán las funciones de ese desarrollo y son elementos indisociables y caras de una misma moneda.

En las consideraciones sobre el alumno bajo este paradigma constructivista, se puede plantear que alumno es visto como un constructor activo de su propio conocimiento. Para los piagetianos es él quien debe actuar en todo momento en el aula escolar. De manera particular, se considera que el tipo de actividades que se debe fomentar en los alumnos son aquellas de tipo auto iniciadas (que emerjan del alumno libremente) las cuales en la mayoría de las ocasiones no pueden ser dejadas a la espontaneidad en la enseñanza de lenguas extranjeras.

El constructivismo comenzó a modificarse con el descubrimiento de la obra de Lev Vygotsky que refleja la importancia de la ayuda en las relaciones sociales para el aprendizaje en un momento histórico con que muestra una cultura determinada y Michel Foucault quien realizó su crítica, centrado en las relaciones de poder, a constructivistas como Paulo Freire, constructivismo en la situación cultural, y Jürgen Habermas, constructivismo en la comunicación entre los hombres. 
Rev. SINAPSIS, Vol. 3, N² 2, Diciembre 2013

ISSN $1390-7832$

Para Foucault "el conocimiento está intrínsecamente ligado con el poder". Este filósofo francés describe los campos del conocimiento como tipos de discursos que consiste en teorías, prácticas, actitudes y hábitos, los cuales no tienen que estar generalmente impuestos de una forma de pensamiento y comportamiento aceptable en la sociedad, deben ser según reglas de poder. Por supuesto, este poder se refleja en el uso de la lengua con que se llega al conocimiento.

De acuerdo con el paradigma histórico - cultural de L. S. Vigotsky, el aprendizaje es aquel proceso en el cual el individuo asimila determinada experiencia histórico cultural, al mismo tiempo que se apropia de ella, por eso es que el aprendizaje bueno es aquel que precede al desarrollo. Esto por supuesto, necesita de un sujeto activo, que le dé sentido a esta experiencia, transformándola en su subjetividad; o sea que el aprendizaje es "un proceso que partiendo de lo externo, del medio como fuente proveedora, se realiza por y en el individuo atendiendo a sus necesidades a través de la actividad y la comunicación propias, y de los otros como portadores estos últimos, de toda la riqueza individual y social” Febles (1992:217)

Lo histórico- cultural, propone que el individuo construye su conocimiento, no porque sea una función natural de su cerebro, sino porque literalmente se le ha enseñado a construir a través de un diálogo continuo con otros seres humanos. No es que el individuo piense y de ahí construye, sino que piensa, comunica lo que ha pensado, confronta con otros sus ideas y luego construye. En esta variante la construcción mental de significados es altamente improbable si no existe el andamiaje externo dado por un agente social. Picket, M. (2000)

Bajo postulados históricos culturales el proceso de enseñanza y aprendizaje se analiza como un único proceso. Álvarez de Zayas (1995), en su libro La escuela en la vida, sustenta que el aprendizaje es la actividad que desarrolla el estudiante para aprender, para asimilar la materia de estudio. La enseñanza es, a su vez, la actividad que ejecuta el profesor. Haciendo un análisis en el proceso docente - educativo tradicional y durante la actividad de enseñanza se considera al estudiante como objeto. En consecuencia sobre él recae la actividad del profesor para que aprenda. El aprendizaje es el resultado y el proceso que dirige el profesor en la enseñanza que tiene en la materia de estudio lo que se aprende y enseña. 
Rev. SINAPSIS, Vol. 3, N² 2, Diciembre 2013

ISSN $1390-7832$

Esta caracterización del proceso mediante estos componentes es inacabada y refleja en muy poca medida sus características más importantes, reduciendo el papel del estudiante a un elemental objeto, sin destacar lo más trascendente, que el alumno es el sujeto de su propio aprendizaje.

Para los conductistas el aprendizaje está condicionado por la respuesta individual a un estímulo, para los cognitivistas el aprendizaje es el resultado de la aplicación individual de los procesos mentales, para los constructivistas es el resultado de la construcción del significado y para los socio históricos el aprendizaje es el producto de la experiencia compartida en situaciones de interacción social. Todas estas posiciones se presentan en el proceso de enseñanza aprendizaje del inglés como lengua extranjera en la universidad, aunque siempre prevalece un paradigma.

En particular, el aprendizaje de lenguas extranjeras ha sido definido de varias formas, para Ann Hammond es: “, es un tipo de instrucción donde los estudiantes trabajan juntos en pequeños grupos para alcanzar el objetivo" (2009: 18) que aunque puntualiza en la instrucción que realiza el estudiante y que lo hace de forma conjunta con otros para lograr objetivos, no particulariza en otros elementos importantes.

Según Eric M. Anderman y Lynley H. Anderman en su libro Psychology of Classroom Learning. An Encyclopedia plantean que el aprendizaje de una lengua es complejo, no importa si adquirido como primera lengua en la infancia, o después en la vida como segunda lengua o lengua extranjera, porque "el proceso consiste en adquirir un sistema de la lengua, en vez de una serie de componentes desconectados. Es un sistema no solo de reglas gramaticales y vocabulario sino la forma adecuada de su uso..." (542) Por eso para los autores de este trabajo se entiende por aprendizaje de una lengua extranjera al desarrollo de un sistema complejo y completo de la lengua en alguna etapa de la vida en que los sujetos se comunican en determinada situación social en una lengua que no es la materna. Este aprendizaje por supuesto se diferencia de la adquisición y tiene lugar en las aulas con la cooperación y el intercambio entre los estudiantes y el profesor.

Fundamenta Johnson y Johnson (1999) que el aprendizaje de lenguas es un aprendizaje cooperativo, que tiene numerosas variaciones, aunque indica cinco características de un aprendizaje cooperativo exitoso:

a) Los estudiantes aprenden que su éxito depende del trabajo en equipo. 
Rev. SINAPSIS, Vol. 3, Nº 2, Diciembre 2013

ISSN $1390-7832$

b) Los estudiantes son individualmente responsables, mientras que el logro de los objetivos es del grupo.

c) Los estudiantes apoyan el trabajo interactivo.

d) Los estudiantes desarrollan habilidades sociales mediante el trabajo cooperativo.

e) Los estudiantes como grupo tienen la oportunidad de reflexionar sobre la eficacia de trabajar juntos.

El trabajo cooperativo crea un buen ambiente donde los estudiantes aprenden el idioma y desarrollan simultáneamente sus competencias en la interacción social con un propósito definido por la sociedad en la que se desenvuelve.

Por otra parte, en lenguas extranjeras, se reconoce como diferente la adquisición y el aprendizaje a partir de los postulados de S. Krashen, para él la adquisición consiste en un proceso espontáneo de internalización de reglas de forma natural en la relación con el lenguaje, mientras que el aprendizaje es el desarrollo del conocimiento consciente de una lengua extranjera a través de un estudio formal. Krashen (1985). Por tanto, para aprender una lengua extranjera se debe hacer uso de estrategias de aprendizaje porque no ocurre la espontaneidad en la internalización de las reglas que ocurre con el sistema de la lengua materna.

Tarone (1983), citado en Lessard-Clouston, (1997), define las estrategias de aprendizaje de una lengua extranjera como "un intento por desarrollar la competencia lingüística y sociolingüística en la lengua de llegada para incorporar estas dentro de su competencia interlingual." Para Rubin (1987) citado en Lessard- Clouston 1997), las estrategias de aprendizaje son "estrategias que contribuyen al desarrollo del sistema de la lengua que el aprendiz construye y afectan directamente el aprendizaje”. O’Malley y Chamot (1990:1) definen las estrategias como "pensamientos o comportamientos especiales que los individuos usan para ayudarse, aprender o retener nueva información"

La definición que aporta Rebeca Oxford, resulta la más completa y útil para el trabajo con una lengua extranjera. Según esta autora las estrategias de aprendizaje de una lengua son “....acciones específicas, comportamientos, pasos o técnicas que los estudiantes (con frecuencia de manera intencional) utilizan para mejorar su progreso en el desarrollo de sus habilidades en la lengua extranjera". Y sigue planteando que: "Estas estrategias pueden facilitar la internalización, el 
Rev. SINAPSIS, Vol. 3, Nº 2, Diciembre 2013

ISSN 1390 - 7832

almacenamiento, la recuperación o el uso de la nueva lengua. Las estrategias son herramientas necesarias para el desarrollo de habilidades comunicativas: Oxford (1990: 18)

En el aprendizaje de una lengua extranjera se ha demostrado que, para lograr un buen nivel de competencia en la L2, es necesario tener mucho contacto con el idioma de un modo constante. Los alumnos necesitan tener acceso directo a la comunicación hablada, preferentemente dentro de un contexto interactivo en el que puedan obtener mucha información sobre la estructura y el funcionamiento de la lengua extranjera. En tales circunstancias los alumnos pueden verificar las hipótesis que se están formando sobre el idioma que están aprendiendo. El aprendizaje de una segunda lengua es un proceso largo y por tanto ha de ser motivado. El alumno tiene que pasar necesariamente por diferentes fases del conocimiento antes de dominar el sistema de la lengua extranjera, sobre todo cuando lo hace para contribuir al aprendizaje de su profesión.

En el programa de la disciplina idioma inglés para la carrera de Comercio Exterior, se explica que cuando se verifica que un estudiante no ha vinculado el idioma extranjero a sus estudios, se comprueba que no sólo limita su caudal informativo y cultural sino como consecuencia su propio desarrollo como futuro profesional. La base del aprendizaje no es el contenido de una lección, sino el proceso de interacción en el aula el que genera las oportunidades de aprender contenidos de la especialidad y de interés individual de los profesionales en formación que contribuyan a la formación integral.

Por consiguiente, la permanencia del idioma inglés durante los seis semestres de la carrera, y la incorporación de otras formas de organización docente, partiendo de reuniones periódicas, no sólo con los profesores del área sino con los de la carrera, para analizar y llegar a consensos de actividades relacionadas con la utilización del idioma, hace que se constituyan esas actividades en componentes esenciales de la interdisciplinariedad en la formación del profesional.

Por otra parte, esta forma de vinculación entre el aprendizaje del inglés con el aprendizaje de otras asignaturas en su formación hace que los profesores contribuyan con su esfuerzo para la creación y edición de libros de texto de especialidad para las carreras, acorde con el perfil del profesional, pero también con los requerimientos del aprendizaje de la lengua extranjera y las tareas a cumplir. 
Rev. SINAPSIS, Vol. 3, No 2, Diciembre 2013

ISSN 1390 - 7832

La tarea comunicativa va a llevar a los alumnos al cumplimiento de los objetivos planteados y al fin máximo de que se logre la comunicación en lengua extranjera como parte de su preparación para la profesión. Por eso la tarea comunicativa constituye la célula fundamental del proceso de enseñanza y aprendizaje de esta lengua extranjera cuyo objetivo final será la competencia comunicativa en inglés. Las tareas comunicativas, además son una vía material de incorporación de la realidad a la clase, o sea de enriquecimiento cultural, a la vez que favorecen el protagonismo del profesional en formación le brindan la oportunidad de utilizar los conocimientos que posee, provenientes de otras materias o de la realidad circundante, para la solución, ya sea en el actuar personal o colectivo, de los retos que la tarea le impone a cada momento.

Por ello el acceso a la información a través del idioma inglés es un proceso que exige: Conocimientos básicos de gramática y vocabulario del sistema lingüístico, uso de los conceptos de la profesión en las funciones comunicativas, conocimiento acerca de cómo los recursos de la lengua se emplean para expresar las funciones comunicativas en el estilo científico propio de la profesión (con generalizaciones, definiciones, clasificaciones, hipótesis, descripciones, argumentos, entre otros) para manipular la información en los diferentes tipos de discursos académicos.

De esta forma el aprendizaje del inglés se inscribe en el accionar pedagógico para lograr competencias. El enfoque por competencia se sustenta por la caracterización de principios como:

1. Los programas de formación son organizados a partir de competencias para aprender.

2. Las competencias varían en función del contexto en el cual están aplicadas.

3. Las competencias están descritas en términos de resultados y normas.

4. Los representantes del mundo del trabajo participan en el proceso de elaboración.

5. Las competencias son evaluadas a partir de los resultados y normas que las componen.

6. La formación tiene un alto contenido práctico.

La Educación en Ecuador en las actuales condiciones del desarrollo económico-social, debe preparar al futuro profesional, con un dominio de saberes y competencias profesionales que 
Rev. SINAPSIS, Vol. 3, N² 2, Diciembre 2013

ISSN $1390-7832$

asegure las bases de su cultura y de su preparación para el trabajo, con habilidades y cualidades personales que le permitan el trabajo en equipo, y adoptar una posición activa y reflexiva en las diferentes actividades en que se desempeñe, tanto en la escuela y el hogar, como en la sociedad en general.

\section{Metodología}

Dentro de los métodos del nivel teórico, se aplica: el método hipotético- deductivo para deducir predicciones empíricas que luego se someten a verificaciones a partir de los datos iniciales; el método de análisis histórico y lógico para estudiar los referentes teóricos que caracterizan el proceso de enseñanza aprendizaje del inglés; los métodos de análisis-síntesis y de induccióndeducción, permiten el análisis y las generalizaciones necesarias sobre el objeto de estudio y su campo de acción para ofrecer solución al problema enunciado; el método sistémico estructural funcional para la elaboración del modelo teórico del proceso de enseñanza aprendizaje del inglés en los estudiantes de la carrera de Comercio Exterior y la estrategia didáctica y el método de la modelación para describir y develar las conexiones entre los componentes del objeto de estudio actual para el desarrollo del proceso de enseñanza aprendizaje con fines específicos.

Los métodos del nivel empírico empleados permiten la recopilación de datos sobre el comportamiento de los fenómenos, objetos y procesos de la realidad educativa específica para dar cumplimiento al objetivo propuesto. Para ello se utilizan: la observación directa para registrar el comportamiento de aspectos e indicadores sobre proceso de enseñanza aprendizaje del inglés y hacer una valoración sobre las formas de enseñanza de las habilidades orales y escritas del inglés

con fines específicos, la entrevista para obtener información de los docentes implicados en la enseñanza de la asignatura Inglés y hacer una valoración de las posibles causas que afectan el proceso de enseñanza aprendizaje del inglés de los estudiantes, y la encuesta con su cuestionario a los estudiantes para evaluar el aprendizaje en este nivel de enseñanza. Además, se aplica el preexperimento para analizar la efectividad del modelo teórico y de la estrategia. 
Rev. SINAPSIS, Vol. 3, No 2, Diciembre 2013

ISSN 1390 - 7832

Dentro de los métodos estadístico-matemáticos, se utilizan el análisis porcentual y la prueba de rangos señalados y pares igualados de Wilcoxon en el procesamiento de los datos obtenidos y en el establecimiento de las generalizaciones correspondientes durante el pre experimento. Y para la aplicación del criterio de expertos se emplea el método Delphi.

\section{Conclusiones}

El objetivo supremo de la enseñanza de idiomas en el mundo es el desarrollo de la competencia comunicativa que ha de ser entendida como la capacidad de usar la lengua para expresar nociones y funciones de comunicación, que se dan a través de las necesidades de uso de la lengua, donde los alumnos son el centro de las actividades.

El proceso de enseñanza aprendizaje de una lengua extranjera debe estar destinado a desarrollar la habilidad comunicativa de los alumnos,

La actividad de aprendizaje de los estudiantes es una cuestión que ha cobrado renovado interés a raíz de los nuevos enfoques de enseñanza- aprendizaje basados en competencias que se están extendiendo en todos los niveles y etapas de los sistemas educativos.

\section{Bibliografía}

1. Álvarez de Zayas, C. (1999) La escuela en la vida. Didáctica. Editorial Pueblo y Educación. La Habana.

2. Anderman E. M. y L. H. Anderman (2009) Psychology of Classroom Learning. An Encyclopedia. Macmillan Social Science Library. New York.

3. Antich de León, R. (1988). Metodología de la enseñanza de las lenguas extranjeras. Ciudad de la Habana: Ed. Pueblo y Educación.

4. Ausubel, D. P. (1975) Psicología educativa. Un punto de vista cognoscitivo. México. Trillas.

5. Brown. (1994) Principles of Language Learning and Teaching ( $3^{\text {rd }}$ ed.) New Jersey: Prentice Hall.

6. Bruner, J. (1960) The Process of Education. Cambridge, MA: Harvard University Press.

7. Bruner, J. (1966) Toward a Theory of Instruction. Cambridge, MA: Harvard University Press. 
Rev. SINAPSIS, Vol. 3, N² 2, Diciembre 2013

ISSN $1390-7832$

8. Bruner, J. (1996) The Culture of Education. Cambridge, MA: Harvard University Press.

9. Chaplin, J. P. y Krawiec, T.S. (1978) Psicología: sistemas y teorías. México: Interamericana.

10. Danserau. (1985) Learning strategy research. In J.W. Segal, S.F. Chipman, \& R. Glaser (Eds.), Thinking and Learning Skills: Relating Learning to Basic Research (pp. 209-240). Hillsdale, NJ: Erlbaum.

11. De Vega, M. (1984) Introducción a la psicología cognitiva, Madrid: Alianza.

12. Díaz F. y Hernández, G. (1998) Estrategias docentes para un aprendizaje significativo. México. Editorial Mc Graw Hill.

13. Febles Elejalde, M. M. (1999) Un punto de vista sobre el carácter activo del sujeto del aprendizaje. En revista Cubana de Psicología. Vol. 16. N 3 . pp. 214.- 221.

14. Foucault, M. (1977) Discipline and Punish: The Birth of the Prison. London: Penguin Books Ltd.

15. Freire, P. (1970) Pedagogy of the Oppressed. New York: Continuum.

16. Gardner, H. (1987) La nueva ciencia de la mente: Historia de la psicología cognitiva. Barcelona: Paidós.

17. Habermas, J. (1992) Further reflections on the public sphere, in C. Calhoun (ed.) Habermas and the Public Sphere. Cambridge, MA: MIT, 421-61.

18. Hammond, Byrd, Anne. (2009) Learning to learn cooperatively. Number 4. English Teaching Forum.

19. Hilgard. E.R. (1968) Teorías del aprendizaje. México Trillas.

20. Hismanoglou. (2000) Languages Learning Strategies in Foreign language Learning and Teaching" En The internet TESL Journal. Vol.V. $\quad N^{\circ} 8$ http://attech.ac.jp./Ites/j/Articles/Hismanoglu-Strategies html.2000

21. Lafrancesco. V. Giovanni. (2004) Evaluación integral de aprendizajes. Taller. Universidad de Antioquia. Abril 29 y 30.

22. Johnsn y Johnson. (1999)Making cooperative learning work. Theory Into Practice 38 (2): 67-73.

23. Krashen. S.(1985) The Input Hypothesis: Issues and Implications, New York: Longman. 
ISSN $1390-7832$

Rev. SINAPSIS, Vol. 3, N² 2, Diciembre 2013

24. Lessard Clouston, M. (1997) Language Learning Strategies: An Overview for L2 Teachers. En The Internet TESL Journal. Vol. III. $\mathrm{N}^{\circ}$ 12, http:// aitech.ac.jp/aiteslj/Articles/lessard- Clouston.html.

25. Mallart. J. (2012) Aprendizaje de Competencias Básicas en Educación Plurilingüe.

26. Monereo. (1994) Estrategias de enseñanza aprendizaje. Formación del profesorado y aplicación en el aula. España: Editorial Graó.

27. Neisser, U. (1982) Procesos cognitivos y realidad. Madrid.

28. O’Malley y Chamot. (1990) Learning Strategies in Second Language Acquisition. Cambridge: Cambridge University Press.

29. Oxford Rebeca . (1990) Styles, strategies, and aptitude: Connections for language learning. In T.S. Parry \& C.W. Stansfield (Eds.), Language Aptitude Reconsidered (pp. 67-125). Englewood Cliffs, NJ: Prentice Hall.

30. Oxford Rebeca. (1993) Language learning strategies in a nutshell: Update and ESL suggestions. TESOL Journal, 2(2), 18-22.

31. Picket. M. D. (2000) The Sleeping Giant: Investigations in Business English. Language International.

32. Pozo, J. I. (1989) Teorías cognitivas del aprendizaje. Madrid: Morata.

33. Riviere, A. (1987) El sujeto de la psicología cognitiva. Madrid: Alianza.

34. Rubinstein, S.L. (1974) El desarrollo de la psicología. Principios y métodos. Buenos Aires: Grijalbo.

35. Weinsten y Mayer. (1986) The teaching of learning strategies. In M.C. Wittrock (Ed.), Handbook of Research on Teaching, 3rd Edition (pp. 315-327). New York: Macmillan.

36. Yaroshevsky, M.G. (1979) La psicología del Siglo XX. México: Grijalbo. 